\title{
Actividad Antimicrobiana de Diversos Aceites Esenciales en Bacterias Benéficas, Patógenas y Alterantes de Alimentos
}

\section{Antimicrobial activity of several essential oils in beneficial, pathogenic and foodborne bacteria}

\section{María Belén Vignola}

Departamento de Ingeniería Química, Facultad Regional San Francisco, CONICET. Av. de la Universidad 501, Córdoba - Argentina

belenvignola@agro.unc.edu.ar

\section{Mónica Serra}

Departamento de Ingeniería Química, Facultad Regional San Francisco

Av. de la Universidad 501, Córdoba - Argentina

monicaserra@hotmail.com

\section{Alfonsina Ester Andreatta}

Departamento de Ingeniería Química, Facultad Regional San Francisco, CONICET. Av. de la Universidad 501, Córdoba - Argentina

aandreatta@sanfrancisco.utn.edu.ar

\section{RESUMEN}

El presente estudio evaluó la actividad antimicrobiana de diferentes aceites esenciales (limón, naranja, mandarina, laurel, eucalipto y burro) en bacterias benéficas como Lactobacillus plantarum ES147 y ATCC 8014, patógenas como Escherichia coli ATCC 25922 y una bacteria alterante de alimentos, Leuconostoc mesenteroides MS1. En los aceites que se registraron halos de inhibición significativos (al menos $10 \mathrm{~mm}$ de halo en 3 cepas analizadas) se procedió a determinar las concentraciones mínimas inhibitoria y bactericida. Los aceites esenciales de limón, eucalipto y burro mostraron un efecto bactericida en todas las cepas analizadas. Los aceites esenciales de naranja y mandarina presentaron muy bajo o 
nulo poder antimicrobiano en las cepas en estudio. Los aceites esenciales de laurel y burro generaron halos de inhibición significativamente más bajos en bacterias benéficas en comparación con las bacterias patógenas o alterantes de alimentos. Dichos aceites pueden considerarse para su adición en films o recubrimientos de alimentos.

Palabras claves: aceites esenciales, actividad antimicrobiana, Lactobacillus plantarum, Leuconostoc mesenteroides, Escherichia coli.

\begin{abstract}
:
The present study evaluated the antimicrobial activity of different essential oils (lemon, orange, mandarin, laurel, eucalyptus and burro) in beneficial bacteria such as Lactobacillus plantarum ES147 and ATCC 8014, pathogens such as Escherichia coli and a food-altering bacterium, Leuconostoc mesenteroides MS1. Subsequently, the minimal inhibitory concentrations and minimal bactericidal concentrations of the essential oils that had a significant bactericidal effect (at least $10 \mathrm{~mm}$ inhibition zone in tree strains) were determined. Lemon, eucalyptus and burro essential oils showed a bactericidal effect in all analyzed strains. Mandarin and orange essential oils presented very low or null antimicrobial inhibition in the strains under study. Laurel and burro essential oils generated significantly lower inhibition halos in beneficial bacteria compared to pathogenic or food-altering bacteria. Such essential oils can be considered for addition in films or food coatings.
\end{abstract}

Keywords: essential oils, antimicrobial activity, Lactobacillus plantarum, Leuconostoc mesenteroides, Escherichia coli.

\title{
INTRODUCCIÓN
}

La seguridad alimentaria es un tópico cada vez más importante, independientemente de los avances en la salud pública y en la conservación de productos alimenticios. Los últimos brotes de enfermedades transmitidas por alimentos han impulsado la búsqueda de formas innovadoras para inhibir el crecimiento microbiano en alimentos (Hernández-Ochoa et al., 2011). Entre las alternativas naturales se encuentran los aceites esenciales (AE) que son productos obtenidos por extracción de materias primas vegetales, en los que se hallan concentrados sabores y aromas característicos. Están constituidos por mezclas complejas de hidrocarburos, compuestos oxigenados y residuos no volátiles. Los AE están contenidos en glándulas o vesículas secretoras inmersas en los tejidos de las hojas, flores, cáscara y semillas de los frutos de muchas especies (Dabbah et al., 1970).

Los fitoquímicos presentes en los $\mathrm{AE}$ han demostrado que son efectivos en una variedad de aplicaciones al disminuir el crecimiento y la supervivencia de los microorganismos transmitidos por los alimentos, convirtiéndolos así, en alternativas adecuadas a los antibióticos (Ruiz-Navajas et al., 2012; Viuda-Martos et al., 2008). Varios investigadores han propuesto que la acción antimicrobiana de los $\mathrm{AE}$ se puede atribuir a su capacidad de penetrar a través de las membranas bacterianas en el interior de la célula y exhibir actividad inhibitoria sobre las propiedades funcionales de la célula y sus propiedades lipofílicas. Soković et al. (2010) aislaron y testearon aceites esenciales de 10 hierbas comúnmente consumidas contra bacterias alterantes de alimentos tales como Escherichia coli (ATCC 0157:H7), Pseudomonas aeruginosa (ATCC 27853), Salmonella enteritidis (ATCC13076), Staphylococcus aureus (ATCC 25923), 
entre otras, y encontraron que el aceite esencial más efectivo fue el de orégano (Origanum vulgare). Asimismo, el trabajo de Callaway et al. (2011) demostró que los aceites esenciales derivados de cítricos eran efectivos para inhibir el crecimiento de bacterias alterantes de alimentos como Escherichia coli, Salmonella Typhimurium, Staphylococcus aureus, Listeria monocytogenes, Campylobacter y otras. Los estudios sobre el efecto de los AE contra una amplia gama de microorganismos, incluida la microflora patógena y de descomposición de alimentos, se encuentran entre las principales perspectivas para una producción segura de alimentos (Trombetta et al., 2005). El objetivo del presente trabajo fue analizar la actividad antimicrobiana de diferentes aceites esenciales en bacterias benéficas como Lactobacillus plantarum ES147 y ATCC 8014, patógenas como Escherichia coli ATCC 25922 y una bacteria alterante de alimentos, Leuconostoc mesenteroides MS1.

\section{MATERIALES Y MÉTODOS}

\section{Material vegetal y extracción de aceites esenciales}

Se utilizaron cáscaras de limón (Citrus lemon), de naranja (Citrus sinensis), de mandarina (Citrus reticulata) obtenidas en el mercado local y hojas secas de laurel (Laurus nobilis), eucalipto (Eucalyptus cinerea) y burro (Aloysia polystachya) recolectadas en territorio del departamento San Justo, de la provincia de Córdoba. Las cáscaras se extrajeron manualmente y se molieron, tanto como las hojas, en un molinillo (Moulinex, $750 \mathrm{~W}$ ) a máxima velocidad por 2 minutos. Los aceites esenciales de limón (AEL), naranja (AEN), mandarina (AEM), laurel (AELa), eucalipto (AEE) y burro (AEB) se obtuvieron mediante arrastre con vapor de agua con un equipo de escala semilaboratorio (Figmay, Argentina), por $90 \mathrm{~min}$ desde que comenzó la condensación. Los aceites esenciales se mantuvieron en un frasco color caramelo y se almacenaron a $4^{\circ} \mathrm{C}$ hasta su uso. La composición de cada aceite fue descripta previamente por Serra et al. (2018).

\section{Cepas bacterianas}

Se utilizaron 4 cepas bacterianas: Lactobacillus plantarum ES147 (aislada de cereales) (Salvucci et al., 2016) y Lactobacillus plantarum ATCC 8014 como cepas benéficas, Escherichia coli ATCC 25922 como cepa patógena y Leuconostoc mesenteroides MS1 aislada a partir de salchicha de Viena (Serra et al., 2018) como cepa alterante de alimentos. Ambas cepas Lactobacillus plantarum (ES147 y ATCC 8014) y Leuconostoc mesenteroides MS1 crecieron en caldo MRS (de Man, Rogosa y Sharpe) a $30^{\circ} \mathrm{C}$ por $24 \mathrm{~h}$ y $48 \mathrm{~h}$ respectivamente, mientras que Escherichia coli ATCC 25922 creció en caldo tripteína soya a $37^{\circ} \mathrm{C}$ por $24 \mathrm{~h}$.

\section{Análisis de sensibilidad}

Las pruebas de sensibilidad se llevaron a cabo con los diferentes extractos naturales mediante el método de difusión en agar (Malbrán, 2012). El inóculo se preparó siguiendo el método de suspensión directa de colonias en una solución salina $(\mathrm{NaCl}$ al 8,5\%), para obtener una densidad de 0,5 en la escala de Mc Farland, que se corresponde, aproximadamente, con una concentración de 1,5 x $108 \mathrm{UFC} / \mathrm{ml}$. La inoculación se realizó con un hisopo embebido en esta solución estandarizada, que se extendió uniformemente en el medio de cultivo agarizado. Se impregnaron discos de papel de filtro estéril (125 mm, Munktell) de $5 \mathrm{~mm}$ de diámetro con $10 \mu \mathrm{l}$ de cada AE y se colocaron 3 discos en cada placa. Luego, las placas de Petri se incubaron a $30^{\circ} \mathrm{C}$ durante $24 \mathrm{~h}$ para Lactobacillus plantarum ES147 y Lactobacillus plantarum ATCC 8014; a $30^{\circ} \mathrm{C}$ durante $48 \mathrm{~h}$ para Leuconostoc mesenteroides MS1 y a $37^{\circ} \mathrm{C}$ durante $24 \mathrm{~h}$ para 
Escherichia coli ATCC 25922. El aceite esencial difunde desde el disco al medio de cultivo, produciendo un halo de inhibición, en los casos en que éste posea propiedades antimicrobianas. En los casos en que hubo inhibición, el proceso continuó tomando una muestra del halo de inhibición, inoculándolo en agar correspondiente a cada cepa e incubándolo a la temperatura y tiempo correcto. Cuando no hubo crecimiento, el antimicrobiano fue considerado de naturaleza bactericida mientras que, si hubo crecimiento, se consideró que el antimicrobiano era de naturaleza inhibitoria. Se usó un disco de gatifloxacina (Gatif Forte, Poen, $0.5 \% \mathrm{p} / \mathrm{p}$ ) como control positivo y un disco de lecitina de soja granulada (Modelife, $70 \% \mathrm{p} / \mathrm{p}$ ), como control negativo (no presenta actividad antimicrobiana). Los diámetros de los halos de inhibición se midieron con un calibre.

\section{Determinación de Concentración Mínima Bactericida (CMB) y Concentración Mínima Inhibitoria (CMI)}

La concentración mínima inhibitoria (CMI) se define como la concentración más baja de un antimicrobiano que inhibe el crecimiento visible de un microorganismo después de su incubación. La concentración mínima bactericida (CMB) se considera la concentración más baja de antimicrobiano que evita el crecimiento de un organismo después del subcultivo en medios libres de antibióticos (Andrews, 2002). En el presente trabajo se determinó la CMI y $\mathrm{CMB}$ de los aceites esenciales que formaron halos de inhibición significativos (halo mayor a $10 \mathrm{~mm}$ en al menos 3 cepas analizadas), utilizando un método de macrodilución en caldo (Cockerill et al., 2012). Se preparó una solución madre de cada AE que contenía $1 \mathrm{ml}$ de aceite $+5 \mathrm{ml}$ de lecitina de soja $(2 \% \mathrm{p} / \mathrm{p})$ para formar una emulsión. Cada tubo se inoculó con una asada de la cepa a analizar, preparado como se describió anteriormente, para lograr una concentración inicial de 1,5 x $108 \mathrm{UFC} / \mathrm{ml}$. Los tubos se incubaron a temperatura y tiempo correspondientes a cada cepa, junto con un tubo de control sin AE. La CMI se estableció observando el crecimiento (turbidez en el medio de cultivo) en los distintos tubos después de incubarlos; mientras que para la determinación de la CMB se procedió a tomar una asada de los tubos en los que no se detectó turbidez y extenderla en la superficie de placas con agar, para luego incubarlas en las condiciones específicas para cada cepa.

\section{Análisis estadístico}

Se utilizó el programa InfoStat (Di Rienzo et al., 2018). Se realizó un análisis de la varianza (ANOVA, $a=0,05$ ) y el test DGC (Di Rienzo, Gusman y Casanoves) para determinar diferencias significativas entre las medias de los halos de inhibición.

\section{Resultados y discusión}

\section{Análisis de sensibilidad}

Se observó una gran variación en las propiedades antimicrobianas de los diferentes aceites esenciales en estudio. El diámetro del halo de inhibición se encontró en el rango de 6-19 mm para todas las cepas analizadas (Tabla 1). Los AEE y AEB presentaron mayor actividad antimicrobiana contra todas las cepas analizadas, con diámetros de halos de inhibición medio de 12,17 $\mathrm{mm}$ y 11,83 $\mathrm{mm}$ respectivamente. El AEL presentó moderada actividad contra todas las cepas analizadas con diámetro promedios de inhibición de 9,66. El AEN registró el menor poder antimicrobiano, con un diámetro promedio de halo de inhibición de 6,33 $\mathrm{mm}$, e inhibiendo sólo a las cepas Lactobacillus plantarum ES147 y Escherichia coli ATCC 25922. 
Estos resultados están en concordancia con Fernández-López et al. (2005) quienes también encontraron al AEN inefectivo contra Leuconostoc mesenteroides subsp. mesenteroides 824 . Ambrosio et al. (2017) encontraron resultados similares de diámetro de inhibición del AEL (7,6 mm) y AEN (7,2 mm) contra Lactobacillus plantarum ATCC 8014, aunque el halo de inhibición registrado en dicha cepa por el AEE fue mucho menor al registrado en el presente trabajo $(8 \mathrm{~mm})$.

\begin{tabular}{|l|c|c|c|c|c|c|}
\hline & Limón & Naranja & Mandarina & Laurel & Eucalipto & Burro \\
\hline $\begin{array}{l}\text { Lactobacillus } \\
\text { plantarum } \\
\text { ES147 }\end{array}$ & $10,00 \pm 3,40 \mathrm{~b}$ & $7,17 \pm 0,98 \mathrm{a}$ & $7,67 \pm 0,52 \mathrm{a}$ & $\mathrm{ni}$ & $13,00 \pm 2,65 \mathrm{a}$ & $7,67 \pm 0,58 \mathrm{a}$ \\
\hline $\begin{array}{l}\text { Lactobacillus } \\
\text { plantarum } \\
\text { ATCC } 8014\end{array}$ & $7,17 \pm 2,56 \mathrm{~b}$ & $\mathrm{ni}$ & $6,67 \pm 0,82 \mathrm{a}$ & $11,00 \pm 1,00 \mathrm{a}$ & $14,33 \pm 0,58 \mathrm{a}$ & $8,33 \pm 0,58 \mathrm{a}$ \\
\hline $\begin{array}{l}\text { Escherichia coli } \\
\text { ATCC 25922 }\end{array}$ & $14,50 \pm 3,15 \mathrm{c}$ & $5,50 \pm 0,63 \mathrm{a}$ & $6,50 \pm 0,55 \mathrm{a}$ & $18,33 \pm 0,58 \mathrm{c}$ & $11,33 \pm 1,53 \mathrm{a}$ & $16,67 \pm 1,53 \mathrm{~b}$ \\
\hline $\begin{array}{l}\text { Leuconostoc } \\
\text { mesenteroides } \\
\text { MS1 }\end{array}$ & $7,00 \pm 2,28 \mathrm{a}$ & $\mathrm{ni}$ & $\mathrm{ni}$ & $13,00 \pm 1,00 \mathrm{~b}$ & $10,00 \pm 2,00 \mathrm{a}$ & $14,67 \pm 3,06 \mathrm{~b}$ \\
\hline
\end{tabular}

a. Diámetro de halo de inhibición incluyendo el disco de $5 \mathrm{~mm}$, expresados como el promedio de 3 réplicas

b. ni: no inhibió

c. Letras diferentes en la misma columna indican diferencias significativas entre las cepas analizadas $(P<0,05)$.

Tabla 1. Diámetros de halos de inhibición ( $\mathrm{mm}$ ) de AE sobre cepas analizadas a,b,c

La existencia de diferencias en la actividad antimicrobiana reportada de los aceites esenciales de la misma planta, puede deberse a sus diferentes orígenes geográficos, temporadas de cosecha, genotipo, clima, procedimiento de secado y la parte destilada de la planta, entre otras. Toda esta variabilidad influye en la composición química y la concentración relativa de cada componente en los aceites esenciales (Oussalah et al., 2007).

El AELa y AEB fueron más efectivos para inhibir Escherichia coli ATCC 25922 que al resto de las bacterias tal como se observa en los diámetros de inhibición de 18,33 mm y 16,67 mm respectivamente. El AELa generó significativamente mayor halo de inhibición en Escherichia coli ATCC 25922 que en el resto de las cepas; mientras que el AEN contra Escherichia coli ATCC 25922 solo generó un halo de 5,5 mm de promedio. Resultados similares fueron encontrados por Guo et al. (2018) quienes estudiaron diferentes aceites esenciales de cítricos y encontraron resistencia antimicrobiana para Escherichia coli ATCC 25922 con el AEL $(11,1 \mathrm{~mm})$ y el AEN (7,01 mm), en concordancia con los resultados de este trabajo. La acción de aceites esenciales de cítricos contra bacterias patógenas fue demostrada por Fisher y Phillips (2008) quienes reportaron una fuerte actividad antimicrobiana de AEL frente a Escherichia coli ATCC 25922. El AEB presentó significativamente mayor actividad antimicrobiana contra la bacteria patógena (Escherichia coli ATCC 25922) y la alterante de alimentos (Leuconostoc mesenteroides MS1), que contra ambas cepas benéficas (Lactobacillus plantarum ES147 y ATCC 8014).

Se sabe que es necesaria una composición óptima de la microbiota gastrointestinal para la salud intestinal de los seres humanos, lo que permite alcanzar una inmunidad contra 
agentes nocivos como las bacterias patógenas (Choct, 2009). Entre los microorganismos que componen la microbiota gastrointestinal animal, se encuentran las llamadas bacterias beneficiosas o probióticas, como Lactobacillus y Bifidobacterium, cuya acción benéfica es la inhibición de las bacterias patógenas por exclusión competitiva (Sugiharto, 2016). Debido a esto, es deseable que los $\mathrm{AE}$ sean selectivos y ejerzan una acción antibacteriana contra bacterias patógenas y no contra bacterias benéficas del tracto gastrointestinal animal (Ouwehand et al., 2010). En cuanto a los AEN, AEM y AEE no se evidenciaron diferencias significativas en la actividad antimicrobiana entre las cepas analizadas. La mayoría de los estudios que investigan la acción de los AE contra los organismos presentes en los alimentos y los patógenos transmitidos por los alimentos coinciden en que, en general, los AE son ligeramente más activos contra las bacterias Gram-positivas que las Gram-negativas (Delaquis et al., 2002; Oussalah et al., 2007; Smith-Palmer et al., 2001). Los aceites esenciales deberían ser más efectivos contra bacterias Gram-positivas debido a la directa interacción entre la membrana celular con los componentes hidrofóbicos de los AE quienes separan los lípidos de la membrana celular bacteriana y las mitocondrias y, en el proceso, hacen que la célula se vuelva más permeable (Burt, 2004; Friedly et al., 2009). Basado en esta premisa, las bacterias Gramnegativas deberían ser más resistentes a los AE debido a que poseen una pared celular hidrofílica (Calo et al., 2015). Sin embargo, los resultados obtenidos en este trabajo mostraron un mayor diámetro de halos de inhibición en bacterias Gram-negativas (Escherichia coli ATCC 25922), que en las bacterias Gram-positivas (Lactobacillus plantarum ES147, Lactobacillus plantarum ATCC 8014, Leuconostoc mesenteroides MS1) para AEL, AELa y AEB. Se encontraron diferencias significativas entre los diámetros de halos de inhibición para AEL y AELa $(p=0,05)$. Este fenómeno puede explicarse por la existencia de diferentes targets celulares en bacterias que se unen específicamente con diferentes compuestos de distintos modos, lo que permite que incluso las bacterias Gram-negativas sean más sensibles que las bacterias Gram-positivas (Klein et al., 2013). Estos resultados están en concordancia con Deans y Ritchie (1987) quienes concluyeron que las bacterias Gram-positivas y Gram-negativas eran igualmente sensibles a los AE de cítricos como el limón, la mandarina y la naranja.

\section{Determinación de Concentración Mínima Bactericida (CMB) y Concentración Mínima Inhibitoria (CMI)}

Los AE que exhibieron mejores actividades antimicrobianas en el método de difusión en agar (burro, laurel, eucalipto y limón) fueron seleccionados para determinar la CMI y CMB, en cada cepa analizada (Tabla 2).

\begin{tabular}{|l|c|c|c|c|c|c|c|c|}
\hline & \multicolumn{2}{|c|}{ Limón } & \multicolumn{2}{|c|}{ Laurel } & \multicolumn{2}{c|}{ Eucalipto } & \multicolumn{2}{c|}{ Burro } \\
\hline & $\mathrm{CMI}$ & $\mathrm{CMB}$ & $\mathrm{CMI}$ & $\mathrm{CMB}$ & $\mathrm{CMI}$ & $\mathrm{CMB}$ & $\mathrm{CMI}$ & $\mathrm{CMB}$ \\
\hline Lactobacillus plantarum ES147 & $\mathbf{0 , 4 2}$ & $\mathbf{0 , 6 0}$ & sd & sd & $\mathbf{0 , 4 8}$ & $\mathbf{0 , 6 5}$ & $\mathbf{0 , 6 4}$ & $\mathbf{0 , 6 7}$ \\
\hline Lactobacillus plantarum ATCC 8014 & $\mathbf{0 , 4 1}$ & $\mathbf{0 , 5 4}$ & $\mathbf{0 , 5 6}$ & $\mathbf{0 , 6 7}$ & $\mathbf{0 , 4 2 5}$ & $\mathbf{0 , 4 5}$ & $\mathbf{0 , 3 5}$ & 0,44 \\
\hline Escherichia coli ATCC 25922 & $\mathbf{0 , 5 5}$ & $\mathbf{0 , 9 6}$ & $\mathbf{0 , 3 2}$ & $\mathbf{0 , 3 3}$ & $\mathbf{0 , 4 2}$ & $\mathbf{0 , 5 9}$ & $\mathbf{0 , 3 4}$ & $\mathbf{0 , 6 2}$ \\
\hline Leuconostoc mesenteroides MS1 & 1,89 & 2,35 & $\mathbf{0 , 6 4}$ & 1,34 & 0,72 & 0,79 & 0,65 & 0,75 \\
\hline
\end{tabular}

${ }^{a} \mathrm{sd}: \sin$ datos

Tabla 2. Valores de Concentración Mínima Inhibitoria (CMI) y Concentración Mínima Bactericida (CMB) expresados en $\mathrm{mg} / \mathrm{ml}$ de aceite esencial sobre cepas analizadas ${ }^{a}$ 
De la observación de la Tabla 2, podemos decir que los valores de la CMI y CMB estuvieron comprendidos en los rangos de $0,32 \mathrm{mg} / \mathrm{ml}-1,89 \mathrm{mg} / \mathrm{ml}$ y $0,33 \mathrm{mg} / \mathrm{ml}-2,35 \mathrm{mg} / \mathrm{ml}$ respectivamente.

En el caso de la cepa Lactobacillus plantarum ES147 las CMI y CMB del AEB registraron los valores más elevados mientras que el AEL registró los valores más bajos. Para la cepa Lactobacillus plantarum 8014, las CMI y CMB más elevadas se registraron para AELa; mientras que las más bajas se registraron para el AEB. Respecto a la Escherichia coli ATCC 25922 las CMI y CMB más elevadas se presentaron en el AEL, mientras que los valores más bajos se encontraron en AELa. En cambio, para la cepa Leuconostoc mesenteroides MS1 la CMI más baja se observó al utilizar el AELa y la más alta al usar el AEL, mientras que la CMB más baja se registró para el AEB mientras que la más alta para el AEL. Además, podemos decir que el AEL registró la CMI y la CMB más elevada frente a Leuconostoc mesenteroides MS1 mientras que el AELa presentó la CMI y CMB más baja frente a Escherichia coli ATCC 25922.

La actividad antibacteriana de los diversos aceites analizados y reportados en las Tablas 1 y 2 evidencia que, la existencia de altos valores de diámetros de inhibición se refleja en valores bajos de CMI y CMB para todas las cepas analizadas.

\section{Conclusión}

El presente estudio demostró el poder antibacteriano selectivo de los aceites de limón, naranja, mandarina, laurel, eucalipto y burro contra bacterias benéficas (Lactobacillus plantarum ES147 y ATCC 8014), patógenas (Escherichia coli ATCC 25922) y alterante de alimentos (Leuconostoc mesenteroides MS1). La actividad antibacteriana de los diversos aceites analizados que se evidenció en altos valores de diámetros de inhibición se confirmó mediante los bajos valores de CMI y CMB obtenidos en las diversas cepas.

El AEL, AEE y AEB mostraron un efecto bactericida en todas las cepas analizadas. El AEN y AEM presentaron muy bajo o nulo poder antimicrobiano en las cepas en estudio sin presentar diferencias significativas entre las cepas analizadas. El AELa y AEB generaron halos de inhibición significativamente más bajos en bacterias benéficas en comparación con las bacterias patógenas o alterantes de alimentos. Es por ello que dichos AE podrían ser potencialmente utilizados, por ejemplo, en films o recubrimientos comestibles para alargar la vida útil de los alimentos, ya que inhibirían el crecimiento de bacterias alterantes sin inhibir a las bacterias benéficas que nuestro organismo necesita para su correcto funcionamiento. 


\section{REFERENCIAS}

Ambrosio, Carmen M.S., Severino M. de Alencar, Ricardo L.M. de Sousa, Andrea M. Moreno, y Eduardo M. Da Gloria. (2007). "Antimicrobial Activity of Several Essential Oils on Pathogenic and Beneficial Bacteria", Industrial Crops and Products, 97, 128-36.

Andrews, J. M. (2002). "Determination of Minimum Inhibitory Concentrations", Journal of Antimicrobial Chemotherapy, 49, 1049-1049.

Burt, S. (2004). "Essential Oils: Their Antibacterial Properties and Potential Applications in Foods-a Review", International Journal of Food Microbiology, 94, 223-53.

Callaway, T. R., Carroll, J. A., Arthington, J. D., Edrington, T. S., Anderson, R. C., y Ricke, S. C. (2011). "Citrus Products and Their Use Against Bacteria: Potential Health and Cost Benefits", in Nutrients, Dietary Supplements, and Nutriceuticals, ed. by R.R Watson (United States), pp. 277-86

Calo, J. R., Crandall, P. G., O’Bryan, C. A., y Ricke, S. C. (2015). "Essential Oils as Antimicrobials in Food Systems - A Review'", Food Control, 54, 111-19.

Choct, M., (2009). "Managing Gut Health through Nutrition", British Poultry Science, 50, 9-15.

Dabbah, R., Edwards, V. M., y. Moats W. A. (1970). "Antimicrobial Action of Some Citrus Fruit Oils on Selected Food-Borne Bacteria.", Applied Microbiology, 19, $27-31$.

Deans, S. G., y Ritchie, G. (1987). "Antibacterial Properties of Plant Essential Oils", International Journal of Food Microbiology, 5, 165-80.

Delaquis, P. J., Stanich, K., Girard, B. y Mazza, G. (2002). "Antimicrobial Activity of Individual and Mixed Fractions of Dill, Cilantro, Coriander and Eucalyptus Essential Oils", International Journal of Food Microbiology, 74, 101-9.

Fernández-López, J., Zhi, N., Aleson-Carbonell, L., Pérez-Alvarez, J. A. y Kuri, V. (2005). "Antioxidant and Antibacterial Activities of Natural Extracts: Application in Beef Meatballs", Meat Science, 69, 371-80 .

Fisher, K. y Phillips, C. (2008). "Potential Antimicrobial Uses of Essential Oils in Food: Is Citrus the Answer?", Trends in Food Science and Technology, 19, 156-64.

Cockerill, F. R., Wikler, M. A., Alder, J., Dudley, M. N., Eliopoulos, G. M., Ferraro, M. J., Hardy, D. J. y Hecht, D. W. "Performance Standards for Antimicrobial Disk Susceptibility Tests" , Approved Atandard, Clinical and Laboratory Standars Institute - NCCLS, 2012, XXXII

Friedly, E. C., Crandall, P. G., Ricke, S. C., O’Bryan, M. R. C. y Chalova, V. I. (2009). "In Vitro Antilisterial Effects of Citrus Oil Fractions in Combination with Organic Acids", Journal of Food Science, 74

Guo, J. J., Gao, Z. P., Xia, J. I., Ritenour, M. A., Gao, Y. L., y Shan, Y. (2018). "Comparative Analysis of Chemical Composition, Antimicrobial and Antioxidant Activity of Citrus Essential Oils from the Main Cultivated Varieties in China", Lwt, 97, 825-839.

Hernández-Ochoa, L., Gonzales-Gonzales, A., Gutiérrez-Mendez, N., Muñoz-Castellanos, L. N., y Quintero-Ramos, A. (2011). "Estudio de La Actividad Antibacteriana de Películas 
Elaboradas Con Quitosano a Diferentes Pesos Moleculares Incorporando Aceites Esenciales y Extractos de Especias Como Agentes Antimicrobianos", Revista Mexicana de Ingeniera Quimica, 10, 455-63.

Klein, G., Rüben, C. y Upmann, M. (2013). "Antimicrobial Activity of Essential Oil Components against Potential Food Spoilage Microorganisms", Current Microbiology, 67, 200-208.

Malbrán, G. C. (2012). "Metodo De Determinacion De Sensibilidad Antimicrobiana Por Difusion", Servicio Antimicrobiano, 2012, 9-12.

Oussalah, M., Caillet, S., Saucier, L. y Lacroix, M. (2007). "Inhibitory Effects of Selected Plant Essential Oils on the Growth of Four Pathogenic Bacteria: E. Coli O157:H7, Salmonella Typhimurium, Staphylococcus Aureus and Listeria Monocytogenes", Food Control, 18, 414-20.

Ouwehand, A. C., Tiihonen, K., Kettunen, H., Peuranen, S., Schulze, H. y Rautonen, N. (2010). "In Vitro Effects of Essential Oils on Potential Pathogens and Beneficial Members of the Normal Microbiota", Veterinarni Medicina, 55, 71-78.

Di Rienzo, J. A., Casanoves, F., Balzarini, M. G. Gonzalez, L. Tablada, M. y Robledo, C.W. (2018). 'InfoStat Versión 2018’ (Córdoba, Argentina: Grupo InfoStat, FCA, Universidad Nacional de Córdoba).

Ruiz-Navajas, Y., Viuda-Martos, M., Sendra, E., Perez-Alvarez, J. A., y Fernández-López, J. (2012). "Chemical Characterization and Antibacterial Activity of Thymus Moroderi and Thymus Piperella Essential Oils, Two Thymus Endemic Species from Southeast of Spain", Food Control, 27, 294-299.

Salvucci, E., LeBlanc, J. G., y Pérez, G. (2016). "Technological Properties of Lactic Acid Bacteria Isolated from Raw Cereal Material", LWT - Food Science and Technology, 70, 185-191.

Serra, M., Garnero, J. Nicolau, V. y Andreatta, A.E. (2018). "Assessment of Natural Vegetal Extracts in the Inhibition of Leuconostoc Mesenteroides MS1", Agriscientia, 35, 51-62.

Smith-Palmer, A., Stewart, J. y Fyfe, L. (2001). "The Potential Application of Plant Essential Oils as Natural Food Preservatives in Soft Cheese", Food Microbiology, 18, 463-470.

Sokovicx́, M, Glamočlija, J. Marin, P. D., Brkić, D. y Van Griensven, J L D. (2010). "Antibacterial Effects of the Essential Oils of Commonly Consumed Medicinal Herbs Using an in Vitro Model", Molecules, 15, 7532-7546.

Sugiharto, S. (2016). "Role of Nutraceuticals in Gut Health and Growth Performance of Poultry". Journal of the Saudi Society of Agricultural Sciences, 15, 99-111.

Trombetta, D., Castelli, F., Sarpietro, M. G., Venuti, V., Cristani, M., Daniele, M. (2005). "Mechanisms of Antibacterial Action of Three Monoterpenes", Antimicrobial Agents and Chemotherapy, 49, 2474-78

Viuda-Martos, M., Ruiz-Navajas, Y., Fernández-López, J. y Perez-Álvarez, J. (2008). "Antibacterial Activity of Lemon", Journal of Food Safety, 28 , 567-76. 